\title{
The discrimination of structure in vectorgraphs: Local and global effects
}

\author{
TERRY CAELLI \\ University of Newcastle, Newcastle, N.S. W., Australia \\ and \\ PETER DODWELL \\ Queen's University, Kingston, Ontario, Canada
}

\begin{abstract}
Many recent electrophysiological studies have demonstrated specificity for orientation and position tuning in single units of the visual system. Psychophysical investigations have produced complementary evidence for orientation and position coding in vision which is usually interpreted in terms of the underlying neuronal properties. To date, no studies have endeavored to relate such "local" receptive field response profiles to more "global" or field-specific aspects of the input signal. In a series of experiments, we have investigated orientation/position sensitivity as a function of general orientation and position rules governing line element, or "vectorgraph," displays. We have discovered with such vectorgraph images that individual element acuities are determined by the field structures present. These results show that there are specific "top-down" components even to basic orientation/position coding in the visual system.
\end{abstract}

The problem of "stimulus equivalence" was prominent in psychology for many years. As stated by Klüver (1933), Lashley (1942), Pitts and McCulloch (1947), and others, the question in its simplest form is: How is a highly variable, spatially extended stimulus input mapped by the organism into a relatively small number of fixed categories we call patterns? Essentially, what makes a square recognizable as a square, despite variation in size, position, contrast, orientation, etc.? Many solutions have been proposed to this problem, most of them now perhaps mainly of historical interest. The attempted solutions did, however, serve to sharpen up the notion of pattern coding and emphasized the importance of separating the coding processes from the operation of recognition itself. An account of these developments can be found in Dodwell (1970a).

Two very powerful influences have served to remove the question of stimulus equivalence from the forefront of theoretical (and experimental) investigation in recent years. These have been, first, the spectacular discoveries in visual electrophysiology on the coding of visual contours, or pattern elements. This work seems to remove the need to postulate hypo-

This project was partly funded by Grant AOA44 from the Natural Sciences and Engineering Research Council of Canada to the second author and D. W. Muir. The authors would also like to thank Paul Bevan for helping to run these experiments. Both authors are associated with the departments of psychology of their respective institutions. Requests for reprints should be sent to: T. Caelli, Department of Psychology, University of Alberta, Edmonton, Alberta T60 2J9, Canada. thetical coding mechanisms. The second influence stems from more practically oriented research on pattern recognition by machines. Many different schemes have been proposed and successfully implemented for the computer recognition of simple patterns under various degrees of perturbation. As a major theoretical problem, then, the question seems to retain little inherent interest.

However, there are reasons for thinking that the questions about pattern recognition that originally caused psychologists to state the problem of stimulus equivalence need reconsideration. In the first place, despite early enthusiasm for the results of single-unit recording as providing the key to the input code for patterns (e.g., Barlow, Naramishan, \& Rosenfeld, 1972; Dodwell, 1964, 1970; McFarland, 1965), that early promise has not, in fact, been fulfilled. Not only is there disagreement about what single cells in the mammalian visual cortex actually code (De Valois \& De Valois, 1980), there is also rather little experimental or theoretical research on how the coded input is itself processed. To be sure, Julesz and Schumer (1981) were able to claim a strong consensus on the concept of orientation and frequencyspecific channels in early spatial vision. Such channels are defined psychophysically, and we do not question the validity of this approach. However, it stops very far short of answering important questions about how the recognition of patterns is achieved.

We think that it is timely to reconsider the questions that exercised Lashley and his contemporaries, but to rephrase them in modern terms. Many of the recent major advances in vision research, inspired in 
part by the neurophysiological discoveries of Hubel and Wiesel (1962) and others, have considered local processes of image filtering, contour extraction, and the like. What has been largely missing is a treatment of the integrative mechanisms that generate coherent patterns from such locally processed elements. Models for interactive processes that yield field-like effects have been proposed (e.g., Caelli, Preston, \& Howell, 1978; see also Caelli, 1981), but these have, in general, considered "bottom-up" data-driven systems. Also, progress in the field of computer vision (e.g., Marr, 1976; Winston \& Brown, 1979) has shown how the recovery of pattern information can be obtained on the basis of specific computational principles. Neither of these approaches gives explicit recognition to the possibility that there are organizational properties (built into the visual mechanisms) which constrain the local processing of information. This notion was, of course, a primary concern of those who posed the original problem of stimulus equivalence.

We are not concerned here with the sorts of contextual effects that are readily demonstrated in experiments on object recognition, perceptual constancy, etc. Rather, the question is: Can it be demonstrated that the local processing of pattern elements is subject to "top-down" control by global mechanisms built into the visual brain?' ${ }^{1}$ The Gestaltlike flavor of this question is obvious enough. What makes it a modern question, in our view, is the linking of the local processing idea to questions of global organization. We shall make a proposal about the nature of at least one type of global property, and make (testable) conjectures about its effects on the processing of local pattern information. In contrast to the classical Gestalt position that asked general questions about the nature of global properties, we assume their existence and seek to demonstrate their influence on events at the local level. This approach allows us to specify our conjectures quite precisely, and to obtain quantitative estimates of the global effects.

Our main postulate is that the whole-field transformation under which pattern recognition tends to be invariant (that is, in the first place, the transformations of translation, rotation, and dilation; cf. Gibson, 1950; Hoffman, 1966; Dodwell, Note 1) is the framework of one set of global processes. Our principal conjecture is that the field organization implied by those transformations, when manifested clearly in visual patterns, exerts a powerful influence on the positional and (especially) the orientational tuning properties of the local elements of a pattern.

The transformational approach leads us naturally to a vectorfield analysis of visual patterns, and suggests very strongly an appropriate (although not necessary) species of visual pattern to use in our experiments. Field transformations and their associated transformation groups are conveniently specified and embodied in two-dimensional vectorfields, and the representation of such transformations in visual 'vectorgraphs' is immediate (see Figure 1, for example). The framework is also the natural one within which to examine the contrasting characteristics of global (transformational) and local (vector, or lineelement) processing.

The precise definition of what a vectorfield is need not concern us here; a few simple examples convey most of what is required to understand our approach. (More extensive discussions of the concepts of a manifold and its associated vectorfields, without too much mathematical formalism, can be found in Bruter, 1977, and Dodwell, Note 1.) We start with the concept of a tangent vector. This is a short line segment, tangent to a curve-expressed as $y=f(x)$ in the simplest two-dimensional case-and can be interpreted as the instantaneous rate of change in $y$ as $x$ changes, for all values of $x$. The familiar notation for this is $d y / d x$. The function $f(x)$ can also be thought of as being generated by successive tangent vectors, and this idea, when generalized to the generation of all possible curves on a surface by their tangent vectors, expresses the essential nature of a vectorfield.

A vectorfield is thus a rule (or set of rules) whereby a specific orientation (or direction) is associated with each position in the field. Familiar physical examples would be the magnetic field, which is manifested by tapping iron filings on a sheet of paper held over the pole of a magnet, or the surface of a river, where every point on the surface has associated with it a direction and magnitude of current flow.

A vectorfield may have a mathematically defined overall structure. Two simple two-dimensional examples are shown in Figure $2 \mathrm{a}$. There, the tangent vectors to any circle centered on the origin can be expressed by a simple relation between coordinates $x$ and $y$ that is true for all values of $x$ and $y$. The local expression of the structure is given by the orientation of the vector at each particular position, and the global organization, or invariant structure (so obvious perceptually), by the differential equation relating all values of the variables to their rates of change.

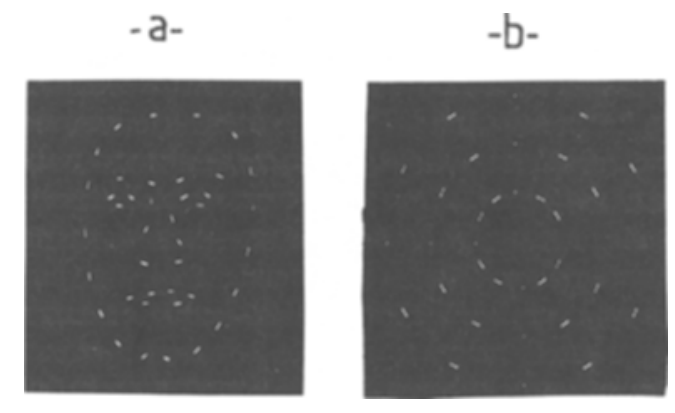

Figure 1. (a) A vectorgraph, which is a set of line elements whose orientation/position $(\phi / P)$ characterlatics define the visual pattern: (b) Rotation-invariant vectorgraph for which the $\phi / P$ remains constant under rotations. 

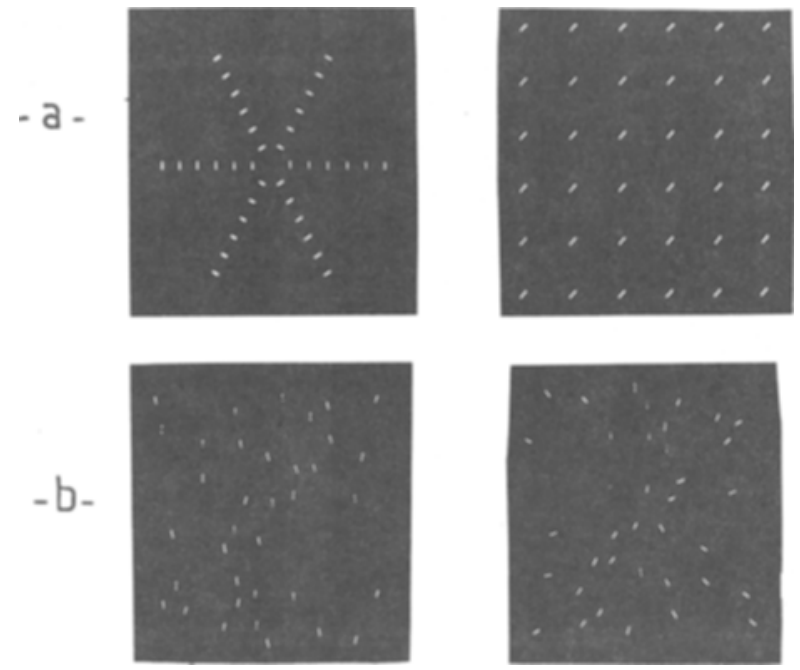

Figure 2. (a) Vectorgraph representations of vectorfields whose $\phi / P$ coding rules do not change under $(\theta$ deg) rotations and ( $a$ unit) translations, respectively. The generators of these fields are: $d y / d x=-x / y$ and $d y / d x=1$ ). (b) Cases in which the codes are not invariant under rotations.

Construing visual processes within such a framework may have heuristic value and be mathematically convenient, but the main reason for doing so is-as the example of Figure 1 surely demonstrates-that such "vectorgraphs," as we have called them, embody very powerful visual effects. Indeed, a relatively small number of oriented lines on a plain field can strongly suggest visual patterns and structures (Caelli, 1976), as the Gestalt psychologists knew. This leads quite naturally to the idea that vectorfields are in some sense fundamental to visual processing, which is the major premise on which our approach is based. What was missing from the Gestalt account was any definite model of how vectorfield operations might work in vision, and how they would influence local visual events (Dodwell, 1981). This is a deficiency we shall remedy.

The idea of vector operators in vision is not new (e.g., Brown \& Voth, 1937), and even the idea that the visual field can be conceived of as a manifold with associated vectorfields was first suggested some time ago (Hoffman, 1966). We are indebted to Hoffman for this idea, and for his treatment of vectorfield structures in the so-called Lie transformation group theory of neuropsychology (LTG/NP), which is very much a "top-down" approach to the analysis of visual patterns. (A recent statement of LTG/NP, and associated commentary by other scientists, can be found in Paillard, 1977; a nontechnical introduction to the theory is in Dodwell, Note 1). We here present experiments that relate vectorfield structures to the psychophysical properties of local contour elements.

We distinguish between a vectorfield and a vectorgraph as follows: A vectorfield has all the properties described earlier, so that every point on a (two- dimensional) surface has an associated direction and magnitude. Potentially any point on the surface can be so characterized. A vectorfield may be described in terms of physical, chemical, physiological, etc., gradients, or by mathematical properties, but obviously the mathematical characteristic is the most abstract and most general. A vectorfield can be manifested in various ways (some of which we have mentioned), an important example being the vectorgraph. Two vectorgraphs can be instances of the same vectorfield, for example, one specified by a particular mathematical rule, but have different numbers of elements in different positions in the two. So long as every element in every array obeys the vectorfield rule, they represent the same vectorfield. Two vectorgraphs, on the other hand, are identical only if each contains identical numbers (and sizes) of elements in identical positions and orientations.

In the experiments reported here, we consider only the question of discriminating between pairs of vectorgraphs that are identical except for a specified local perturbation. A further step would be to study discrimination between different vectorgraphs which represent the same vectorfield but which vary in other ways, such as in element position or density, as well as in local perturbations.

We aim to investigate whether the sensitivity to perturbations of line element position and/or orientation over an array is a function of the field structure of that array. Two vectorgraphs such as those shown in Figure 3 are identical, except that the line elements in Figure $3 \mathrm{~b}$ have each been rotated by a random angle $\theta(0<\theta< \pm 20 \mathrm{deg})$ from the corresponding orientations in Figure 3a. Casual inspection of the two figures is enough to convince one that, although in some sense they both represent the "same" array, there is also a perceptible difference, and that the detectability of this difference will obviously depend on the range of $\theta$. A similar statement could be made for two vectorgraphs in which only position is changed. In other words, local perturbations alone determine the difference between the two vectorgraphs. An appropriate psychophysical experiment will demonstrate the effective tuning of local

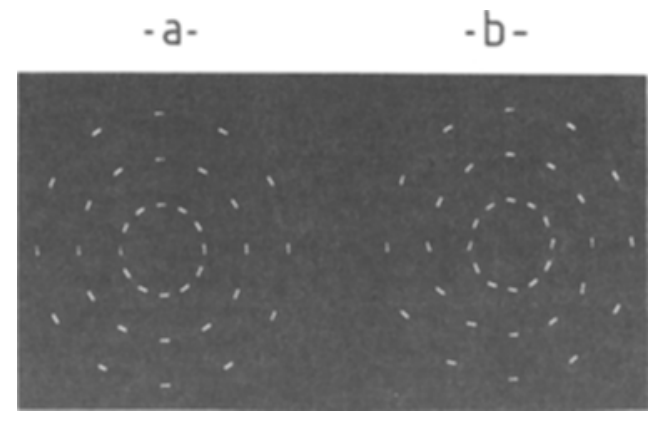

Figure 3. (a) Vectorgraph and (b) random orientation perturbations of \pm 20 deg about each angle in (a). 
elements in such an array. Our objective is to demonstrate that this tuning is a function of field structure, that is, that the ease with which a difference between two patterns like those in Figure 3 can be detected will be a function of the pattern's global properties. Our conjecture is that when the field structure is strong, being, for example, a field with the invariant structure of one of the planar transformation groups discussed, the orientational tuning will be fine, and that when the structure is weak or nonexistent (random, for instance), the tuning will be coarse. To put it simply, when there is clear structure in the vectorgraphs, small perturbations in element orientations will be easily detected, but when the structure is weak or absent, much larger local changes will be required for two vectorgraphs to be seen as different from each other. If such "topdown" or field determinants are not present, then we would expect orientation and position sensitivity to be solely predictable from the number (spatial summation), size, and spatial density of the elements.

\section{EXPERIMENT 1: ORIENTATION TUNING FOR ELEMENTARY FIELDS}

The aim of this initial experiment was to measure the effects of several different field structures on the orientational tuning of the line segments (of fixed length and position) that constitute the elements of a vectorgraph. Since the properties of a structured vectorfield are defined in terms of both position and orientation, specification of one (say, position) determines the value of the other (orientation) for every element. The structure of the field can thus be distorted in two distinct ways: positions can be specified and the associated orientations perturbed, or orientations can be specified and the associated positions perturbed. These are not equivalent operations, and, of course, both perturbations could be made at the same time. In this experiment, we consider orientational tuning only, by asking subjects to make forced-choice "same-different" judgments between pairs of vectorgraphs displayed simultaneously, where the only difference between the pair displayed on a given trial is the random orientational perturbation $(\theta)$ in one of them. Orientational tuning is estimated by measuring discrimination as a function of the random perturbation angle range $( \pm \theta)$.

\section{Method}

Stimuli and Apparatus. The two vectorgraph element properties of position and orientation were both fixed at one of four different states and then combined factorially to yield 16 different vectorgraphs. The states, or characteristics, were: $\mathbf{R}=$ random, $\mathbf{P}=$ parallel, $\mathbf{C}=$ circular, and $\mathbf{S}=$ star. The definitions of these states with respect to position and orientation are as follows: $\mathbf{R}$ position $-n^{2}$ random locations (within a given square); $R$ orientation-random orientation between 0 and $180^{\circ} ; P$ positionsquare position matrix ( $\mathrm{n} \times \mathrm{n}$ locations, fixed increment size); $\mathbf{P}$ orientation-fixed orientations (all $45 \mathrm{deg}$ ); this is an orientation field corresponding to translation invariance; $C$ position-locations generated on concentric circles ( $\mathrm{m}$ circles with $\mathrm{r}$ positions/ circle: $\left.m \times r=n^{2}\right) ; C$ orientation-at any location, orientation was set to that of a tangent to a circle with fixed center (common to all locations); this orientation field corresponds to rotational invariance; $S$ position-locations generated on lines radiating from a common center ( $m$ lines with $r$ positions/line: $m \times r=n^{2}$ ); and $S$ orientation-orientations set to a radiating pattern, orthogonal to $\mathrm{C}$ orientation; this field corresponds to dilation invariance.

We call $R, P, C$, and $S$ (subscripted with $p$ or $\theta$ when ambiguity exists) the position and orientation codes of the vectorgraphs. Each vectorgraph contains $n^{2}=36$ elements, and the factorial combination of the four position $x$ four orientation codes yielded the 16 "basic" vectorgraphs illustrated in Figure 4 . Here we use the symbol $\phi / p$ to represent the orientation/position code mixtures.

For every basic vectorgraph, the orientations were randomly perturbed by $\pm(5,10,15,20,30,45) \mathrm{deg}$, as already illustrated in Figure 3. Since the aim of this experiment was to establish orientation sensitivity as a function of basic vectorgraph type, each one was presented with the six appropriate perturbed versions. These image pairs were generated on an HP 1310A CRT under control of an HP 1350A graphics translator-PDP 11/40 computer system. The luminance of each vector was set at $12 \mathrm{cdm}^{-2}$, each vectorgraph subtending $5.71 \mathrm{deg}$ of visual angle with each vector corresponding to $10 \mathrm{~min}$ of arc.

Subjects. Four observers with normal or corrected-to-normal vision were used. Two knew the hypotheses under investigation and two did not, although one of the latter knew the experimental procedure in detail. All were informed of the composition of trial blocks and the nature of the catch trials.

Procedure. An experimental trial consisted of the presentation of a basic vectorgraph with one of its six perturbed versions. Trials occurred in blocks, a block consisting of 20 presentations of the basic vectorgraph, with each perturbed version presentation order being randomized over perturbations and with respect to left-hand presentation. Within each trial block, 20 catch trials were randomly inserted, a catch trial being the presentation of the $\pm 15 \mathrm{deg}$ perturbed version simultaneously at left and right. There were 16 trial blocks, yielding $16 \times 6 \times 20$ trials, plus $16 \times 20$ catch trials, or 2,240 trials per observer. The order of trial blocks was nonsystematic, and different for each observer. Two sessions were required for each observer to complete his trials.

Pilot runs established that an exposure time of $700 \mathrm{msec}$ was required in order to obtain standard psychometric discrimination functions. This exposure time permitted observers to fixate on each stimulus figure briefly, yet without prolonged scrutiny, before making a decision.

The task was simply to decide, as quickly and accurately as possible, whether the two vectorgraphs were identical or different. That is, observers were asked to judge if all positions and orientations in the two images were the same and to respond accordingly. This strict criterion was employed in order to examine critically the null hypothesis that orientation tuning is not a function of the vectorfields represented within a given vectorgraph.

\section{Results and Discussion}

Figure 5A shows the percentage discrimination data for all 16 vectorgraphs, averaged over the four observers. Best fitting logistic distributions have been used to determine representative psychometric functions. This function is defined by

$$
E(P D)=\frac{e^{p_{1}+p_{2} \theta}}{1+e^{p_{1}+p_{2} \theta}},
$$

where $E(P D)$ corresponds to the expected discrimina- 

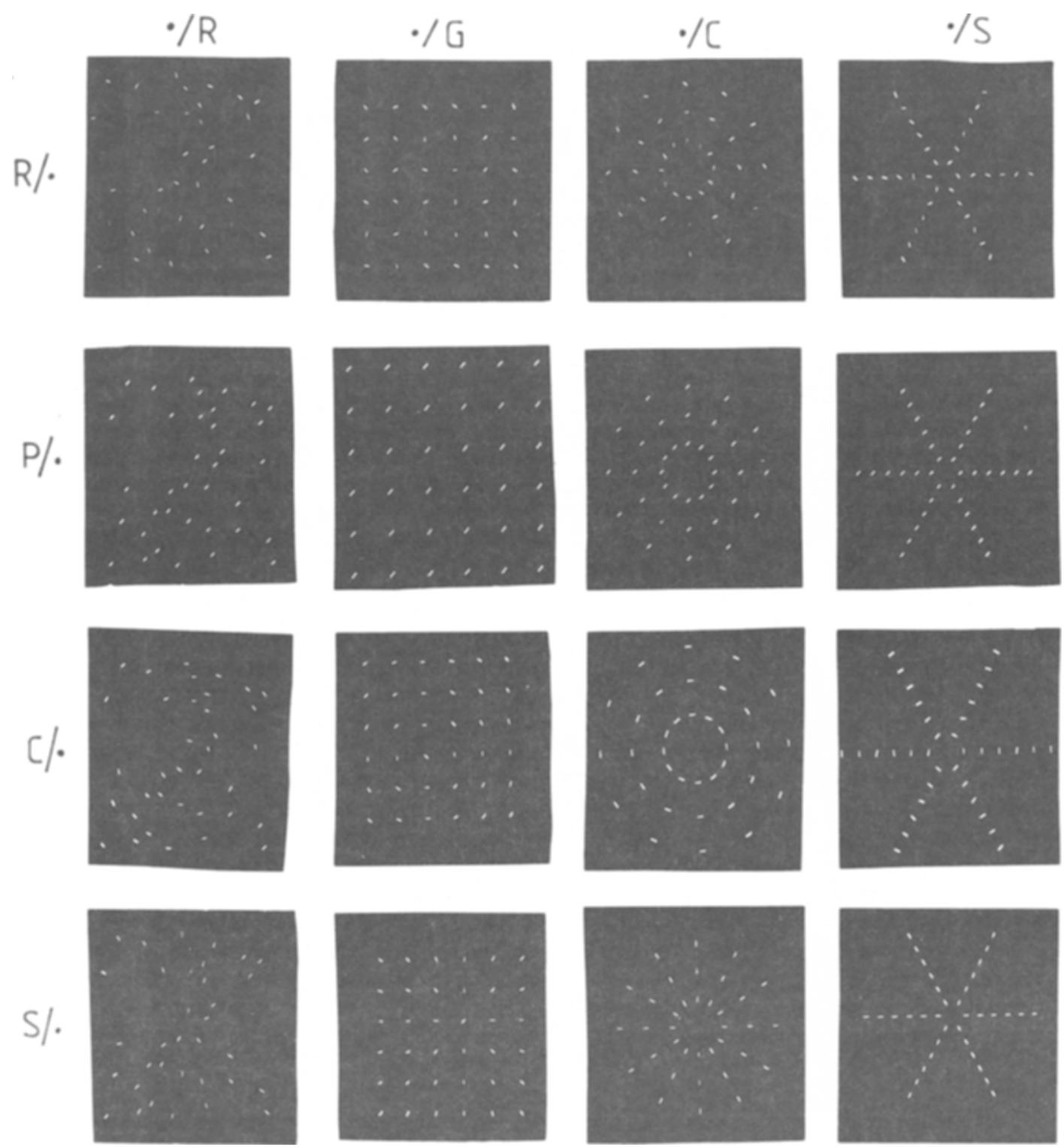

Figure 4. Sxteen basic vectorgraphs used in Experiment 1. Columns indicate the four different position codes $(\cdot / X$, where $X \equiv$ random, grid, circle, star); rows indicate the orientation codes $(Y /$. , where $Y \equiv$ random, parallel, circle, star).

tion probability as a function of the orientation perturbation angle range $(\theta) ; p_{1}, p_{2}$ are model parameters. Except for the random orientation-code cases (row 1 of Figure 5A), the $75 \%$ threshold could be readily estimated, and seemed to discriminate adequately between the various psychometric functions. These individual thresholds were estimated from equivalent psychometric functions determined for each observer on each vectorgraph. In the few cases in which the detection level did not reach $75 \%$, we set an arbitrary threshold at the maximum possible, \pm 45 deg. There were 6 of these (all subjects on $R / R$, two on $R / G$ ), of the total 64 functions. Averages of these thresholds are shown in Figure 5B.
A two-way repeated measures analysis of variance was conducted on the threshold data, yielding significant position $[F(3,9)=5.54, p<.05]$, orientation $[F(3,9)=13.84, p<.05]$, and position $\times$ orientation $[F(9,27)=2.97, p<.05]$ effects. An identical analysis was conducted on the catch-trial data, yielding no significant difference between vectorgraphs (p > .05). The mean error rate for these trials ("different"' response) was $37.9 \%$.

The data show some consistent trends that are easy to interpret and some that are not so straightforward. The major finding is that as the orientation and position codes become structured, orientation sensitivity increases. The overall difference in Figure 5B between 

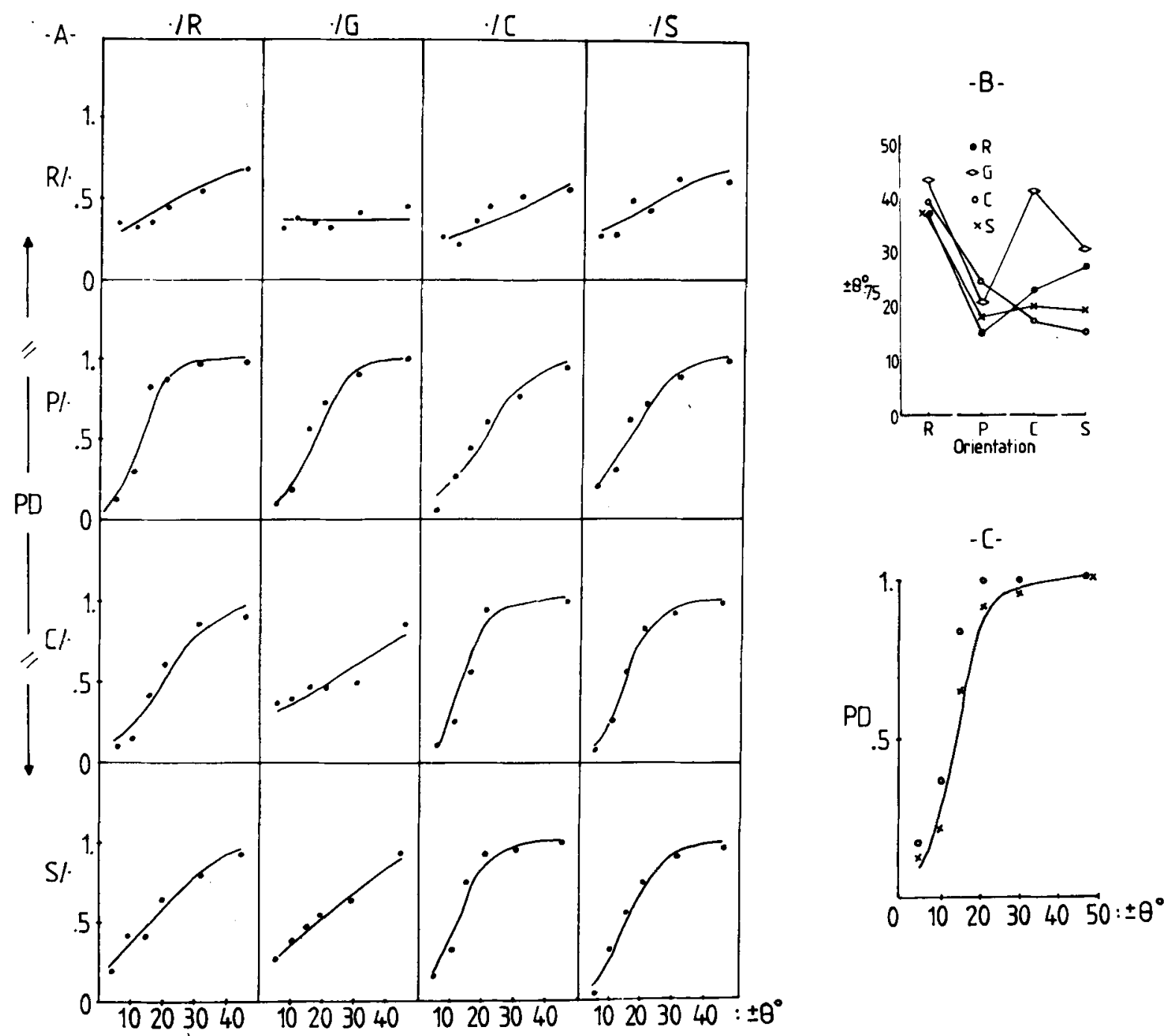

Figure 5. (a) Percentage discrimination (PD) for each vectorgraph in Figure 4 (defined by orientation/position code combinations) as a function of the orientation perturbation range [ $\pm \theta$ (in degrees)] used. Continuous lines correspond to best-fitting logistic distributions. Each point is based on 80 observations over all subjects. (b) 75\% orientation thresholds [ $\pm \theta$. 5 (in degrees)] from (a) over the 16 vectorgraphs. The four orientation codes are shown on the abscissa, and the four position codes are represented by each connected set of points. (c) Psychometric function (continuous curve) for the $C / C$ vectorgraph in Figure 4 [(a) above]. $O$ represents replication with two subjects; $X$ corresponds to average percentage discriminations (PD) for the same two subjects over the orientation perturbations $[ \pm \theta$ (in degrees)] for a $\mathrm{C} / \mathrm{C}$ vectorgraph for which the nearest-neighbor distance is constant for all positions (equal arc increments for all circles).

orientation sensitivity when orientations are random and that when they form part of a structured field is obvious enough. The nearly identical performance over all orientation conditions for the circle and star position codes is remarkable but not surprising since, qua position, the two codes are essentially the same. There is a suggestion in the data that sensitivity is particularly good when the position and orientation codes are "congruent" or correlated (that is, for example, when the $\mathrm{C}$ position and orientation codes are both implemented in the same vectorgraph), the $\mathbf{R}$ codes being the exception, as one would expect.
An anomaly in Figure SB is the case of the $\mathrm{G}$ position (grid) with $\mathbf{C}$ orientation (circular), a condition that yields a surprisingly high orientation discrimination threshold. This might suggest the inhibitory effect of position information on orientation information in certain cases, or perhaps is due to failure of the scanning or processing strategy observers were using for the task. Only further experimentation will decide the issue.

Our major hypothesis, that orientational tuning (sensitivity) is strongly influenced by the amount of structure in the field, is clearly confirmed. 
Can the data be explained in terms of other vectorgraph statistics? For example, could differences in the orientation variances of the basic vectorgraphs account for the differences in tuning? This cannot generally be the case, since vectorgraphs with $C$ and $S$ orientation codes have higher variance than one with a $P$ orientation code; yet, in certain cases (for example, when they are combined with $C$ or $S$ position codes), they yield much better tuning. Orientation variance, when correlated with discrimination performance, explains only $30 \%$ of the variance $(r=.55)$. Evidently, both orientation and position codes are essential for predicting discrimination performance.

Equally, position statistics do not adequately explain position field effects for each orientation code. For example, by determining the mean and variance for the interposition distances and orientations over all elements (the dipole statistics of each vectorgraph position code; see Caelli, 1981), we find that the progression from random to parallel to circular (star) fields yields increasing means and variances on both parameters. Yet, results clearly indicate differential effects of position on orientation codes. Moreover, since dipole statistic differences relate directly to Fourier amplitude spectra differences (see Caelli, 1981), these data indicate that the effects are not due to orientation/frequency energy-distribution differences alone.

Another possibility is that minimum element separation, rather than means and/or variances, might determine local tuning; inspection of Figure 4 shows that the $S$ and $C$ position codes have smaller nearest neighbor distance statistics than the others. To test this possibility, we ran a subsidiary experiment with the $\mathrm{C} / \mathrm{C}$ vectorgraph of Figure 4 and a second version of $\mathrm{C} / \mathrm{C}$ in which the minimum separation was equivalent to that in the $G$ position code. That is, positions were generated on each circle such that the arc length increment was a constant (equal to the square grid vertical/horizontal increment). The psychometric functions obtained for two observers (using the same paradigm as in Experiment 1) gave results virtually identical to the originals. These are shown in Figure SC. Clearly, minimum element separation is not responsible for the superior tuning in $C$ and $S$ vectorgraphs, although, in general, such a relationship between position and orientation codes would seem to be of the utmost importance in determining recognition and discrimination.

We conclude from these results that it is the joint occurrence of specific orientation/position codes that determines orientation sensitivity. The question of whether certain vectorfields have heightened sensitivity because they manifest the structure of transformations of the whole field or simply because they are generally familiar is the subject of the following experiment.

\section{EXPERIMENT 2: ORIENTATION SENSITIVITY WITHIN COMPLEX VECTORGRAPHS}

In this experiment, the aim was to compare orientation discrimination thresholds for a variety of vectorgraphs of familiar and other geometric orientation/position codes. Figure 6 shows the basic vectorgraphs used. For the parallel (square grid) position field, three different orientation codes were used: the vertical $\left(\mathrm{P}_{90}\right)$ and two "smooth" vectorfields generated by two functions $\left[F_{1}(x, y), F_{2}(x, y)\right.$, where $0<(x, y)$ $<2 \pi$ :

$$
\begin{aligned}
& F_{1}(x, y)=\cos (x)-y \\
& F_{2}(x, y)=1-\frac{1}{x+y} .
\end{aligned}
$$

$F_{1}$ and $F_{2}$ determine the orientation code at each position $(x, y)$ in the vectorgraph.

The vertical $\left(\mathbf{P}_{90}\right)$ case was generated as a comparison with $P_{4 s}$, the oblique case studied in Experiment 1 . The special functions $\left(F_{1}, F_{2}\right)$ were used to investigate whether vectorgraphs representing other simple (geometric) rules have orientation sensitivities similar to those already found. These do not correspond to the invariant vectorfields for any oneparameter transformations of the whole field such as were used previously. A random position field with a vertical orientation code $\left(\mathbf{R}_{\mathbf{9 0}}\right)$ was also used to check the position effect on vertical orientation sensitivity.

In addition to these four basic vectorgraphs $\left(\mathbf{P}_{90}\right.$, $F_{1}, F_{2}, R_{90}$ ), five others were generated. The first two consisted of schematic representations, or "vectorsketches," for two more familiar scenes, a face (FA ( $_{0}$ ) and a landscape $\left(\mathrm{SC}_{0}\right)$. The next two consisted of \pm 45 deg random perturbations of these images to give $\mathrm{FA}_{90}$ and $\mathrm{SC}_{90}$. Finally, a circular orientation code was imposed on the face position field (FAC). These latter vectorgraphs were constructed to investigate whether or not orientation tuning on such familiar position fields is affected by the absolute orientations employed-in particular, whether or not congruence between position and orientation codes is critical, or even important.

For all nine cases, orientation perturbations of $\pm(5,10,15,20,30,45)$ deg were used, as in the previous experiment. It should be noted that, for the $\mathbf{F A} \mathbf{A}_{90}$ and $\mathbf{S C}_{90}$ cases, these perturbations were defined on each already distorted, although fixed, position/orientation. Again, 20 catch trials, consisting of duplicates of the \pm 15 deg perturbation, were used, giving $9 \times 140=1,260$ trials per observer. 


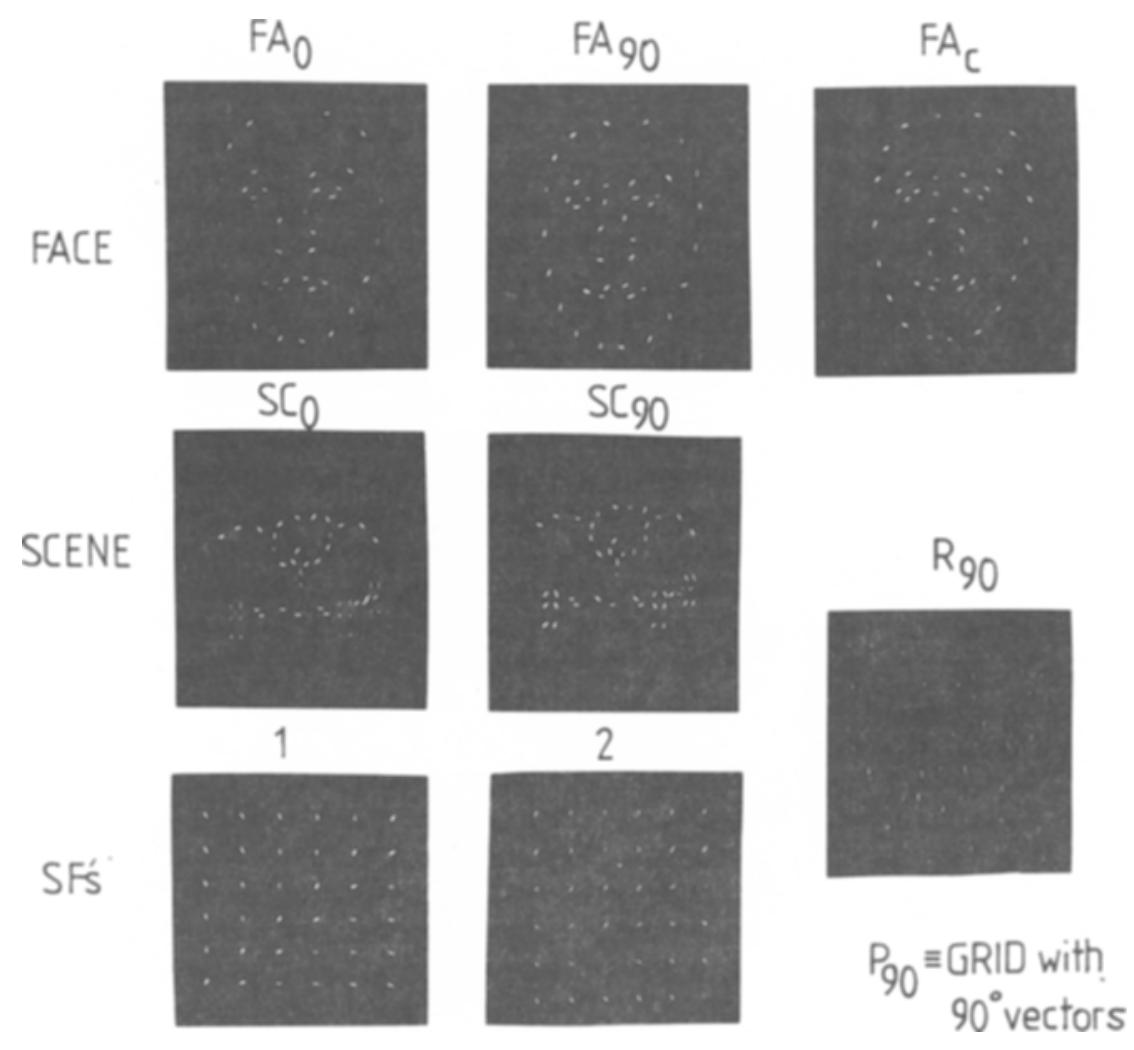

Figure 6. Vectorgraphs used in Experiment 2.

Observers, display conditions, exposure times, and response instructions were identical to those used in Experiment 1.

\section{Results and Discussion}

Figure 7a shows a representative range of vectorgraph discriminations as a function of orientation perturbations. As in the previous experiment, $75 \%$ orientation thresholds were then used in a one-way repeated measures analysis of variance (images $\times$ observations). This yielded a significant main effect $[F(8,24)=6.68, p<.05]$, as shown in Figure 7b. Post hoc analyses (Scheffé tests) showed pairwise significant differences between the special functions $\left(F_{1}, F_{2}\right)$ and the other seven images only. ${ }^{2}$

The main result was that orientation thresholds for the more familiar scene and face images were not as low as for the correlated orientation/position vectorgraphs of Experiment 1. Moreover, the thresholds were not changed by uncorrelating the orientation from the position field (for example, compare $\mathbf{F A}_{90}$, $\mathrm{FA}_{C}$ to $\mathrm{FA}_{0}, \mathrm{SC}_{90}$ to $\mathrm{SC}_{0}$ ). These familiar images have orientation discrimination thresholds somewhere between the correlated fields and the random and "special function" vectorgraphs.

Again, we may ask whether these results can be explained in terms of statistical properties of the vector- graph element distributions. When the nine vectorgraphs are ordered by increasing standard deviations of their orientation distributions (as in Figure 7b), it can readily be seen that there is no monotonic relationship between orientation distribution variances and discrimination sensitivity. Such a relationship would be expected if simple spatial summation occurred independently of the position code. This has been found to be the case in texture discrimination experiments (Caelli, 1981; Caelli \& Julesz, 1978). So Figure $7 \mathrm{~b}$, and the associated analyses, demonstrate that the position code does contribute somewhat to orientation tuning.

However, if position codes are held constant, orientation thresholds should then be monotonically related to orientation variance, given that spatial summation is operating. Relevant data are shown in Figure 7c for both parallel (grid) and random position fields. The monotonicity seems adequately represented by a linear function in both cases, although some nonlinear and asymptotic behavior might be expected. This result suggests that spatial summation, or the accumulation of orientation distribution characteristics over all positions, does operate in this task and can be isolated when identical position codes are used. This does not change the fact that covariation of both codes is important to 
(a)

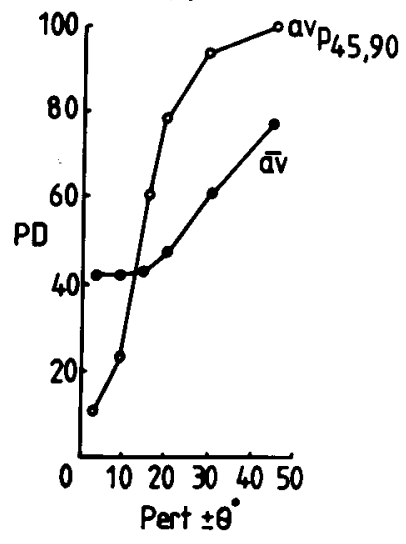

(c)

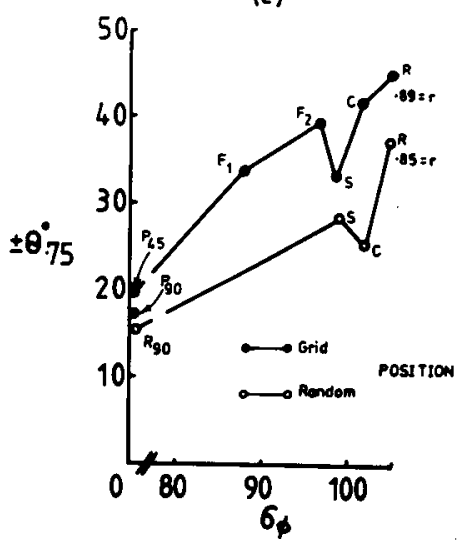

(b)

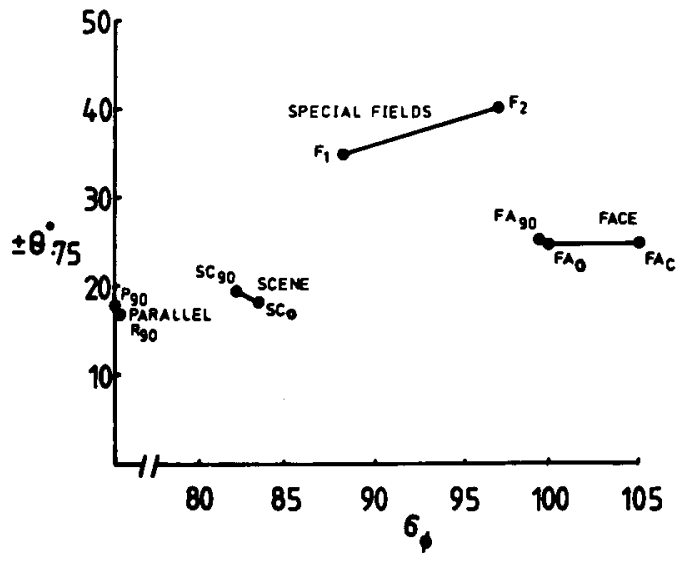

Figure 7. (a) Percentage discrimination (PD) as a function of orientation perturbathon [Pert $\pm \theta$ (in degrees)] for the average $P_{46}, P_{x 0}$ vectorgraphs, and the grand average over all nine images. (b) $75 \%$ orlentation threshold levels $( \pm \theta, . s)$ for the nine images, where Images are plotted as a function of their orientation dbtribution standard devlations $\left(o_{\phi}\right)$. (c) $\pm \theta_{.7}$ levels for all images over the two experiments generated on parallel (aquare grid) and random $(O)$ position fields, plotted againat orlentation distribution standard deviations $\left(0_{4}\right)$. Correlations are shown.

orientation-based vectorgraph discrimination. This latter component has already been demonstrated in Figure 5.

To complement these findings, a third experiment was needed, namely, one in which position sensitivity was measured directly, both as a function of orientation coding and of the imposed field structure.

\section{EXPERIMENT 3: POSITION SENSITIVITY}

In this experiment, two types of position perturbations were investigated: those inducing random position jitters while preserving the vectorfield properties of the orientation value $\left(\mathrm{J}_{\mathrm{I}}\right)$, and those inducing jitters that keep the orientation code of the undistorted position values $\left(J_{P}\right)$. These two cases are shown in Figure 8a; note that the distinction is not relevant to the random and parallel orientation cases. The distinction is important because it is precisely the orientation/position correlation that affects orientation sensitivity.

\section{Method}

Stimulus and Apparatus. Table 1 indicates the cases of parallel $\left(J_{P}\right)$ and invariant $\left(J_{I}\right)$ orientation codes used with the position jitter factor.

As in Experiments 1 and 2, 36-element basic vectorgraphs were used, each vector subtending a visual angle of $10 \mathrm{~min}$. For both $J_{1}$ and $J_{P}$, position jitters of $1,2,3,4,5$, and 6 min of arc were used, the jitter itself being determined by a random position within a circle whose radius was defined by one of these six jitter values about each point. Otherwise, all conditions, including number of catch trials, were the same as in the previous experiments. This gave a total of $140 \times 17=2,380$ trials for each of four observers.

Procedure. As before, the observer's task was to indicate whether or not the two vectorgraphs displayed were identical. Since the two types of jitter were themselves a source of experimental interest, results for each type were recorded separately.

\section{Results and Discussion}

Initially, a two-way repeated measures analysis of 


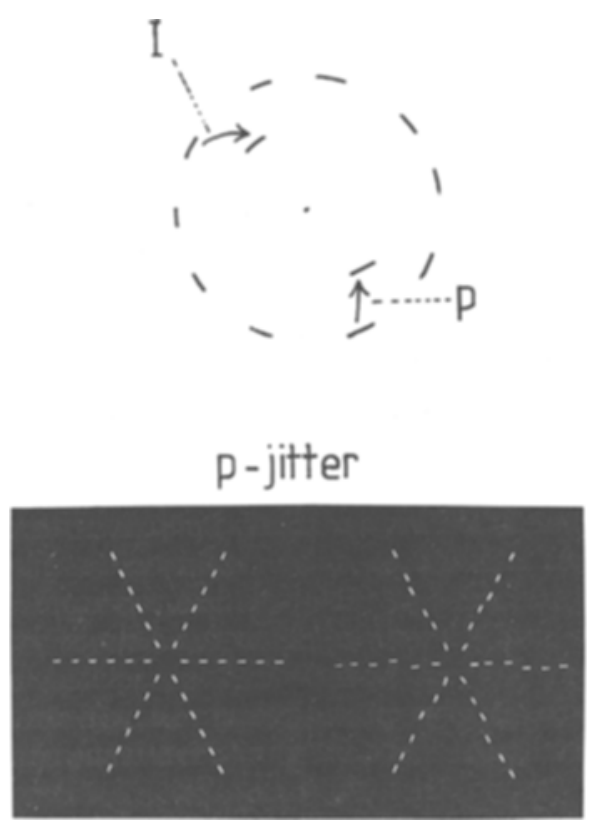

Figure 8. Two types of position perturbation [those in which the orientation is preserved $\left(J_{P}\right.$, where $P=$ parallel) and those which preserve the vectorfield rule $\left(J_{I}\right.$, where $I=$ invariant)] and examples.

variance was computed over the 17 pattern types and six perturbation levels for percentage difference responses over the three observers. Results gave significant pattern $[F(16,32)=6.44, p<.05]$, position perturbation $[F(5,10)=82.14, p<.05]$, and interaction $[F(80,160)=5.26, p<.05]$ effects. Figure 9a shows the average (and individual extrema over all observers and vectorgraphs) discrimination functions for the six perturbation values.

Figure $9 \mathrm{~b}$ shows the mean position perturbation $75 \%$ thresholds estimated from these basic data and observers (here, all $75 \%$ thresholds were below the extreme position jitter range). A three-way repeated measures analysis of variance was computed on the lower half of this threshold matrix (Figure 9b), over position (random, square, circle) $\times$ orientation (circle, star) $\times$ position perturbation type (invariant: $J_{I}$, parallel: $\left.J_{P}\right)$. Position code was the only significant factor $[F(2,4)=127.86, p<.05]$.

Table 1

Vectorgraphs Used in Experiment 3

\begin{tabular}{ccccccc}
\hline \multirow{2}{*}{$\begin{array}{c}\text { Orientation } \\
\text { Code }\end{array}$} & \multicolumn{5}{c}{ Position Code } \\
\cline { 2 - 6 } & & $\mathbf{R}$ & & $\mathbf{P}$ & C \\
\hline $\mathbf{R}$ & $\mathbf{I}$ & - & I & - & I & - \\
$\mathbf{P}$ & - & - & I & - & I & - \\
C & I & P & I & P & I & P \\
S & I & P & I & P & I & P \\
\hline
\end{tabular}

Note $-R=$ random, $P=$ parallel, $C=$ circular, $S=$ square, $I=$ invariant. $J_{I}$ corresponds to position jitters when orientations obey the vectorfield rule; $J_{P}$ corresponds to position jitters that simply keep the original orientation value.
These results clearly demonstrate that, again, the circular fields have field-specific acuities somewhat greater than the square (grid) or random fields with circular or star orientation codes (Figure 5b). Also, this difference is independent of whether the orientation is preserved (parallel) or altered to remain invariant with respect to the field characteristics.

This latter effect seems surprising, but could be due to a tradeoff between two factors that we have already found to affect the sorts of discrimination under investigation. One of these is the nature of the local codes that are manipulated and includes the correlation between the two vectorgraphs. The second factor comprises the global characteristics of the displays. While the parallel $\left(\mathrm{J}_{\mathrm{P}}\right)$ shift produces no local orientation change, orientation within the associated vectorfield becomes misaligned, thereby introducing a discriminable change in the global field. On the other hand, the invariant $\left(\mathrm{J}_{\mathrm{I}}\right)$ shift preserves the vectorfield, and presumably the overall character of the vectorgraph, but the total amount of local change-in both position and orientation codes-is greater than under $\left(J_{P}\right)$ perturbations.

For completeness, we have also observed discrimination under random positional perturbations of the more familiar shapes used in Experiment 2: the face (FA) and scene (SC) vectorgraphs with veridical orientations $\left(\mathrm{FA}_{0}, \mathrm{SC}_{0}\right)$ and $90 \mathrm{deg}$ random perturbations $\left(\mathrm{FA}_{90}, \mathrm{SC}_{90}\right)$, as already used in that experiment. Identical subjects, control conditions, and position perturbation values were used, as with the previous vectorgraphs. In addition, the "special function" $\left(\mathbf{S F}_{2}\right)$ vectorgraph was used as a control image occurring somewhere between the elementary and random fields already studied. Results are shown in Figure 10.

No significant differences were found between the position thresholds for these vectorgraphs, although, in comparison with those in Figure 9, they fall between the thresholds for the random and simple circular and grid fields. These results simply replicate the orientation jitter results supporting the already discussed parallel between orientation and position coding in such vectorgraphs.

\section{GENERAL DISCUSSION AND CONCLUSIONS}

Our main hypothesis, that the tuning of local orientation and position codes can be modulated by global field properties, is clearly supported in all three experiments. The evidence suggests that the effects are rather stronger for orientation than for position codes, although the design of our experiments does not permit a direct comparison of the two.

Our procedure was, in essence, a method-ofconstant-stimuli paradigm, with interspersed "catch" trials. This method suffers an inherent weakness, because response bias is not controlled. However, 
(a)

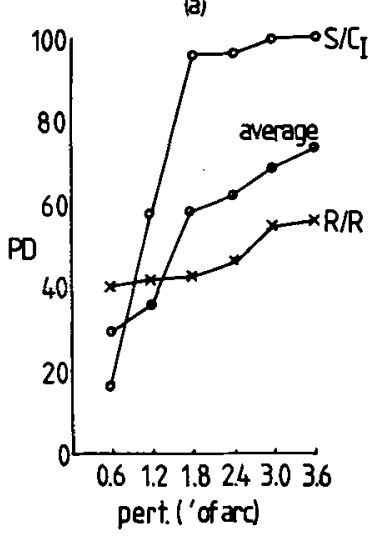

(b)

75\%. THRESHOLDS ["OF ARC]

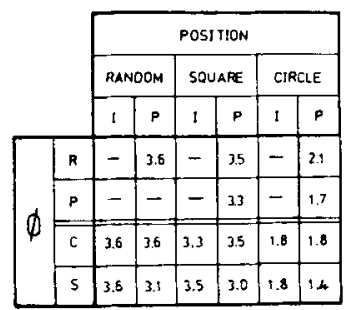

Figure 9. (a) Percentage discrimination (PD) as a function of position perturbation (pert., in minutes of arc) over all vectorgraphs (average) and for the most (atar/circle- $J_{I}$ case: $S / C_{1}$ ) and least (random/random-J $J_{p}$ case) sensitive configurations. (b) Shows $75 \%$ thresholds for discrimination as a function of position fitter for each of the vectorgraphs [defined by position and orlentation $(\theta)$ flelds having elther invariant $\left(J_{I}: I\right)$ or parallel $\left(J_{P}: P\right)$ shifts.]

since we were investigating a new set of phenomena, it seemed sensible to compromise on this point and not use a TSD procedure, in order to investigate such a large number of conditions. The psychometric functions obtained (Figure 5a) look rather good, and are adequately fit by logistic functions. Figure 5c shows that a considerable variation in the vectorgraph element distribution has very little effect on discrimination with the more highly structured vectorgraphs. Whether or not this is true for other displays has not been investigated as yet. Although the conditions of our experiments might have induced a bias toward "different" judgments (as in any constantstimuli design), in fact a reasonable proportion of trials resulted in "same" judgments.

Considering the results of Experiments 1 and 2, we conclude that "optimal" orientation tuning occurs only when specific orientation/position codes correspond to invariant vectorfields for rotations, dilations, and translations. That is, when either position or orientation codes depart from these special cases (Experiment 1), orientation sensitivity decreases. Familiarity is a relevant factor (Experiment 2), as evidenced by the increase in threshold through the familiar shapes to the unfamiliar, special function, then random vectorgraphs (Figure $7 b$ ). We note again that the vectorgraphs with highest orientation sensitivity not only have correlated orientation/ position codes, but also correspond to one-parameter transformations (Caelli, 1981). In comparison, the shapes used in Experiment 2 (except for $\mathbf{P}_{\mathbf{9 0}}, \mathbf{R}_{\mathbf{9 0}}$ ) were compound isoclines, which are not reducible to a set of isomorphic one-parameter transformations.

One can argue that it is the one-parameter nature of the transformations that renders these vectorgraphs special, but it is equally possible that, as in-

stances of linear planar transformations, they have an ecologically special function (Gibson, 1950) that renders their processing unique. Our experiments do not distinguish between these possibilities, and indeed, from a certain theoretical point of view, the two are equivalent (Dodwell, Note 1).

Evidently, the circular organization is particularly salient for both orientation and position codes, in the sense that its modulating effect is strong (Experiments 1 and 3). Similar findings in pattern recognition in kittens (Wilkinson \& Dodwell, 1980) and in color-contingent aftereffects (Emerson, Humphrey, \& Dodwell, Note 2) lead us to conclude that the processing of circularly symmetrical patterns (i.e., those displaying rotational invariance) is especially easy for the visual system. However, we note that, in contrast to the one-parameter $\mathrm{C}$ and $\mathrm{S}$ organizations, our $\mathbf{P}$ codes are ambiguous with respect to translational invariance, since they display no exclusive horizontal or vertical organization. One may speculate that, had we used clearly organized "row" and "column" arrays [as, for example, in anomalous transfer experiments (Dodwell, 1970b)], stronger global effects would have ensued. This point would bear further investigation; we note that the salience of $C$ and $S$ codes in the experiments on pattern recognition and aftereffects referred to was large even when compared with the clear horizontal and vertical organization of grid patterns.

Our findings seem to support and further qualify a two-component model for spatiotemporal contour extraction proposed by the authors (Caelli, 1981; Caelli \& Dodwell, 1980; Dodwell, 1981). The model states that, simply, there are two basic systems operating, one that codes or extracts information-the "bottom-up" system-and one that searches for and

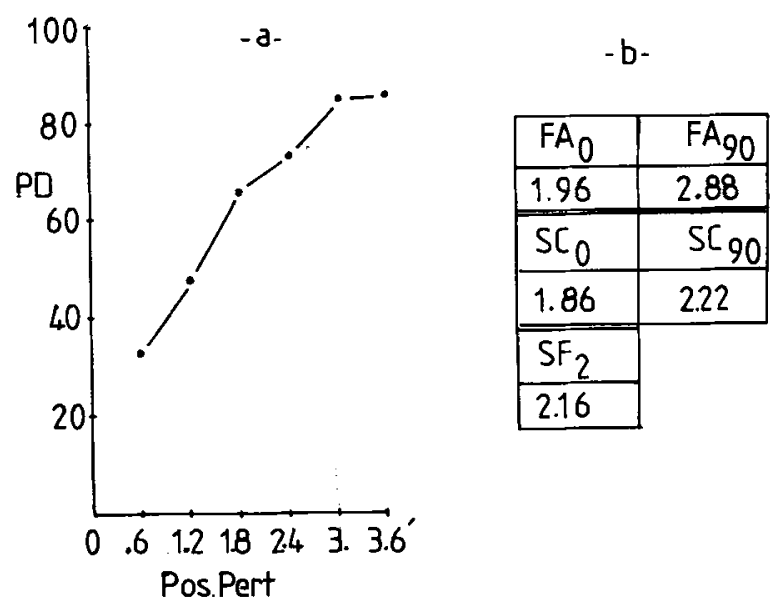

Figure 10. (a) Percentage discrimination (PD), averaged over all five vectorgraphs as a function of position perturbation (Pert, in minutes of are). (b) Shows $75 \%$ position-based diserimination thresholds (Pert, in minutes of arc) for each vectorgraph used. All position jitters were parallel (see text). 
promotes recognition and discrimination of code states under transformations-the "top-down" systems.

The "bottom-up" system has been formulated in terms of spatiotemporal filter profiles that represent the correlated responses of large numbers of receptive fields. In the vectorgraph case, this system operates under spatial summation (i.e., particular parameter states are derived by summation of a retinotopic area) and therefore is nt sensitive to orientation/position conditional constraints as such.

The "top-down" system, in this case, adapts sensitivity precisely as a function of the orientation/ position code constraints, particularly as a function of invariant vectorfields that underlie the vectorgraphs we have used as stimuli. That is, orientation threshold $\left(\theta_{\mathrm{t}}\right.$ : the detectable orientation perturbation range) can be predicted from:

$$
\theta_{\mathrm{t}}=\alpha \sigma_{\phi}-\sum_{\mathrm{j}=1}^{3} \beta_{\mathrm{j}} \operatorname{corr}_{\mathrm{j}}(\phi, P),
$$

where $\sigma_{\phi}$ refers to the standard deviation of the orientation distribution and $\operatorname{corr}(\phi, P)$ corresponds to the correlation between the orientation/position code with respect to transformation $\mathrm{j}$ (one of three: translations, rotations, and dilations). One definition of $\operatorname{corr}_{\mathrm{j}}(\phi, P)$ is:

$$
\operatorname{corr}_{j}(\phi, P)=\sum_{i=1}^{n} \cos \left(P_{i}-P_{k}, \phi_{j}\right),
$$

where $\mathrm{P}_{\mathrm{k}}$ corresponds to the nearest neighbor, or "weighted" position of near-neighbors about $P_{i}$ being on the position field $j$. This quantitative formulation of the two-component model for vectorgraph discrimination will be explored further in a subsequent paper.

Initial estimates from our data suggest that the three $\beta$-transformation coefficients are equally weighted, since introducing correlated $\phi / P$ codes reduced $\theta_{\mathrm{t}}$ values to about the same range in Experiment $1(\approx \pm 15-20$ deg; Figure $7 b)$.

The possibility of inhibitory interaction between position and orientation codes was discussed in conjunction with an apparently anomalous result in Experiment 1. It was found (see Figure 5b) that orientational tuning was surprisingly poor when the $P$ position and $C$ orientation codes were combined. We also raised the possibility that particular sorts of processing strategies might be involved. At a subjective level it is clear to us that different strategies are possible and tend to be employed differently with different vectorgraphs. For example, when $C$ and $P$ orientation codes are used, the strong gestalt of the array seems to "leap out" at the observer in most cases, and the discrimination of vectorgraph differences is made on a holistic basis. In contrast, there are cases-especially the vectorsketch SC-in which processing of any large part of the array within the allotted time becomes impossible. There is, then, a strong tendency to seek out an area within which a particular concatenation of elements (e.g., a small set that happens to be nearly aligned or to follow a circular orbit) yields a distinctive local pattern to be compared with the perturbed version.

This point may seem to vitiate our claim to have distinguished between local and global processes, but we argue that that is not so. We claim that, despite such individual differences in processing strategy, both between vectorgraph types and, no doubt, between individuals, the general effect of global organization upon local feature processing is still clearly in evidence.

What we do concede is that these experiments are but a first attempt to investigate the class of stimulus arrays we have named vectorgraphs, which represent a very rich source for further experimental work. Apart from questions of individual differences just discussed, questions to do with element density, vector length, overall display size, segmented fields, perspective transformations, and nonrandom local perturbations all suggest themselves immediately.

Vectorgraphs not only present a means of investigating organizational factors in vision, but also lend themselves clearly to quantitative treatment. We have demonstrated the salient, and perhaps unique, properties of certain one-parameter transformation groups. These groups play a special role in Hoffman's LTG/NP (Hoffman, 1966), which, we believe, is a useful framework for developing geometrical "topdown" processing ideas. The search for quantitative psychophysical procedures necessary to give substance to that framework was one of the motivations for our experiments.

In these experiments, we have attempted to show that the vectorfield concept is central to an understanding of the recognition of spatially distributed patterns. This concept allows one to formulate global mechanisms for pattern recognition and to model the interactions between the global and local processes. It clarifies the nature of vector operations in the visual field and brings us a step closer to understanding the nature of "stimulus equivalence" as that concept has traditionally been understood.

\section{REFERENCE NOTES}

1. Dodwell, P. C. The Lie transformation group approach to perception. Manuscript submitted for publication, 1982.

2. Emerson, V. F., Humphrey, G. K., \& Dodwell, P. C. Con- 
tingent color aftereffects: Evidence for an integrative mechanism. Manuscript submitted for publication, 1982.

\section{REFERENCES}

Barlow, H. B., Naramishan, R., \& Rosenfeld, A. Visual pattern analysis in machines and animals. Science, 1972, 177, 567-575.

Brown, J., \& Voth, A. The path of seen movement as a function of the vectorfield. American Journal of Psychology, 1937, 49, 543-563.

Bruter, C. P. Initiation à la Géométric différentielle. Cahiers de Psychologie, 1977, 2(3), 73-90.

CaElli, T. M. The prediction of interactions between visual forms by products of Lie operators. Mathematical Biosciences, 1976, 30, 191-205.

Caelli, T. M. Visual perception: Theory and practice. Oxford: Pergamon, 1981.

Caelli, T. M., \& Dodwell, P. C. On the contours of apparent motion: A new perspective on visual space-time. Biological Cybernetics, 1980, 39, 27-35.

Caelli, T. M., \& Julesz, B. On perceptual analyzers underlying visual texture discrimination: Part I. Biological Cybernetics, 1978, 29, 201-214.

Caelli, T. M., Preston, G., \& Howeld, E. Implications of spatial summation models for processes of contour extraction: A geometric perspective. Vision Research, 1978, 18, 723-734.

De Valors, R. L., \& De Valors, K. K. Spatial vision. Annual Review of Psychology, 1980, 31, 309-341.

DoDwELL, P. C. A coupled system for coding and learning in shape discrimination. Psychological Review, 1964, 71, 149-159.

Dodwell, P. C. Anomalous transfer effects after pattern discrimination in rats and squirrels. Journal of Comparative and Physiological Psychology, 1970, 71, 42-51. (a)

DoDWell, P. C. Visual pattern recognition. New York: Holt, Rinehart \& Winston, 1970. (b)

Dodweld, P. C. Geometrical approaches to visual processing. In D. Ingle, R. Mansfield, \& M. Goodale (Eds.), Recent advances in the analysis of visual behavior. Cambridge, Mass: M.I.T. Press, 1981.

Gisson, J. J. The perception of the visual world. Boston: Houghton Mifflin, 1950.
Hoffman, W. C. The Lie algebra of visual perception. Journal of Mathematical Psychology, 1966, 3, 65-98.

Hubel, D. H., \& WIESEL, T. N. Receptive fields, binocular interaction and functional architecture in the cat's visual cortex. Journal of Physiology, 1962, 160, 106-154.

Julesz, B., \& Schumer, R. A. Early spatial vision. Annual Review of Psychology, 1981, 32, 575-627.

KLÜVER, H. Behavior mechanisms in monkeys. Chicago: University of Chicago Press, 1933.

LASHLEY, K. S. The problem of cerebral organization in vision. Biological Symposia, 1942, 7, 301-322.

MarR, D. Early processing of visual information. Philosophical Transactions of the Royal Society, London (series B), 1976, 275, 483-524.

McFarland, J. H. Sequential part presentation: A method of studying visual form perception. British Journal of Psychology, $1965,56,439-446$.

Paillard, J. (Ed.). The Lie transformation group model for perceptual and cognitive psychology. Cahiers de Psychologie, 1977, 2(3), (whole issue).

PitTs, W., \& McCulloch, W. How we know universals; the perception of auditory and visual forms. Bulletin of Mathematical Biophysics, 1947, 9, 127-147.

Wilkinson, F. E., \& Dodwell, P. C. Young kittens can learn complex visual pattern discriminations. Nature, 1980, 384, 258-259.

Winston, P. H., \& Brown, R. H. (Eds.). Understanding vision: Manipulation, computer design, symbol manipulation. Cambridge, Mass: M.I.T. Press, 1979.

\section{NOTES}

1. A further question, but not one we shall address here, is whether such mechanisms-if they exist-are engrained by evolution or acquired by experience.

2. No significant difference was found for catch trials $(p>.05)$, and the overall catch trial error rate was $35.2 \%$.

(Manuscript received February 17, 1982; revision accepted for publication June 11,1982 .) 